\title{
BMJ Open Assessing risks to paediatric patients: conversation analysis of situation awareness in huddle meetings in England
}

\author{
Jacqueline Hayes, ${ }^{1}$ Peter Lachman, ${ }^{\circledR 2,3}$ Julian Edbrooke-Childs, ${ }^{4}$ Emily Stapley, ${ }^{5,6}$ \\ Miranda Wolpert, ${ }^{7}$ Jessica Deighton ${ }^{7}$
}

To cite: Hayes J, Lachman P, Edbrooke-Childs J, et al. Assessing risks to paediatric patients: conversation analysis of situation awareness in huddle meetings in England. BMJ Open 2019;9:e023437. doi:10.1136/ bmjopen-2018-023437

- Prepublication history and additional material for this paper are available online. To view these files, please visit the journal online (http://dx.doi. org/10.1136/bmjopen-2018023437).

Received 1 June 2018 Revised 27 December 2018 Accepted 4 January 2019
Check for updates

(C) Author(s) (or their employer(s)) 2019. Re-use permitted under CC BY-NC. No commercial re-use. See rights and permissions. Published by BMJ.

For numbered affiliations see end of article.

Correspondence to Dr Peter Lachman; plachman@isqua.org

\section{ABSTRACT}

Objectives To analyse the language and conversation used in huddles to gain a deeper understanding of exactly how huddles proceed in practice and to examine the methods by which staff members identify at-risk patients. Setting Paediatric wards in four English hospitals, which were part of a 12-hospital cohort participating in the Situation Awareness for Everyone programme. Wards varied by geographical region and type of hospital. Participants Paediatric staff on wards in four English hospitals.

Design Ethnomethodology and conversation analysis of recorded safety huddles.

Methods This study represents the first analysis of huddle interaction. All huddle meetings taking place on four wards across four different hospitals were audio recorded and transcribed. The research question examined was: how are staff identifying at-risk patients in huddles? The ethnomethodological conversation analytic approach was used to analyse the transcripts.

Results Huddlers made use of categories that allowed them to efficiently identify patients for each other as needing increased attention. Lexicon included the use of 'no concerns', 'the one to watch', 'watcher' and 'acute concerns'. Huddlers used the meetings to go beyond standardised indicators of risk to identify relative risk and movement in patients towards deterioration, relative to the last huddle meeting and to their usual practices. An implicit category, termed here 'pre-concerns', was used by staff to identify such in-between states. Sequential analysis also highlighted the conversational rights that were held implicitly by staff in different clinical roles. Conclusion Practical implications and recommendations for huddlers are considered. These included that for increased situation awareness, it is recommended that all staff are active in the huddle conversation and not only the most senior team members.

\section{INTRODUCTION}

The development of real-time situation awareness (SA) requires a review of a current situation and anticipation of a future state with the creation of solutions before problems happen. Based on the processes of other high
Strengths and limitations of this study

- This study is the first to inductively investigate the categories and methods that staff used in huddles to identify risks to patients.

- Systematic analysis of verbatim transcripts was undertaken to identify precisely how the new intervention progressed and language changes in real-life hospital settings.

- The study identifies the evolution of terminology and interactions between staff.

- Data consisted of audio recordings which has the advantage of capturing huddles in situ rather than in abstraction.

- Some of these recordings were of poorer quality and video recordings capturing non-verbal elements of communication would have enhanced analysis and findings.

reliability industries, for example, the military, nuclear power, aviation and aerospace, huddles have been adopted in healthcare. ${ }^{12}$ $\mathrm{SA}$ in healthcare refers to a shared awareness about a patient's health situation in real and future time. This has implications for organisational hierarchies, as staff members are encouraged to speak about risks without deference to authority.

Huddles are rapid, regular meetings attended by all who may have information about patients and are intended to be non-hierarchical so that all are encouraged to speak or challenge decisions. Participants assess the current state and anticipate future risks to patients, so that the risk can be addressed. ${ }^{1-4}$ The implementation of huddles is correlated with improved patient safety. ${ }^{1}$ Qualitative work suggests that the technique improves organisational efficiency, quality of information sharing, accountability and teamworking culture. ${ }^{23}$ Provost $e t a l^{3}$ conclude that huddles had a decisive impact on improving staff conversation, relationships and culture. 
There has not been any analysis of exactly how huddles proceed in practice at identifying patients at high risk of deterioration, and this is the focus of this article.

Ethnomethodology and conversation analysis (EMCA) studies have examined the practical organisation of meetings at work. This includes topics such as how agendas are managed, employed and strayed from, ${ }^{5}$ how roles are invoked in decision-making processes in multidisciplinary teams, ${ }^{6}$ how decisions are made in teams ${ }^{57}$ and how interprofessional collaboration works in healthcare settings. ${ }^{89}$ The method has been used to highlight important social-interactional moves in the accomplishment of medical tasks. ${ }^{10-12}$ In pulmonary medicine, Chatwin et $a l^{13}$ noted the importance of medical staff providing 'narrative slots' in which patients could provide new information about potentially serious symptoms. In paediatrics, Stivers ${ }^{14}$ showed how through silence, questions and refusal to engage in shared laughter, parents resisted the treatment proposals of doctors who recommended against the use of antibiotics for viral infections. A study of four intensive care unit wards in Italy showed how nurses used detailed and updated information that they had about patients to carefully contribute to medical decision-making in morning briefings. ${ }^{8}$ These enquiries demonstrate that what is said or not said at specific moments in medical conversations can influence the treatment that a patient receives.

A systematic review of clinical handovers in hospitals concluded that there exists a pervasive problem of poor communication during handovers, and that this is leading to error. ${ }^{15}$ Identified problems also included a lack of formal systems for handovers such as a regular designated time and place or a formal obligation to attend. ${ }^{15}$ Eggins and Slade ${ }^{16}$ investigated the discourse of shift handovers. They demonstrated the interdependence between the informational and interactional elements of effective handovers. To improve safety, it is not just what is said, but how it is said and how others receive this information that makes a handover effective.

Huddles, in theory, share many features with handovers in that they involve information sharing, aim for continuity of care and may involve a transfer of accountability when at the end of a shift. The time pressure involved in both situations makes effective communication imperative. However, huddles are theoretically different insofar as they should involve all of those caring for a child (rather than doctors only), focus on at-risk patients and situations rather than all patients, and include anticipation of the future.

The data for this study were taken from a wider evaluation of the Situation Awareness for Everyone (SAFE) safety improvement collaborative. ${ }^{17}$ As part of SAFE, paediatric staff at an initial 12 (Wave 1) then a further 16 (Wave 2) National Health Service hospitals across England trialled several techniques, including huddles, to improve patient care and the anticipation of risks to patients. The aim of this study is to examine the methods by which staff members identify at-risk patients.

\section{METHOD}

\section{Sampling}

A mixed methods approach was taken to the evaluation of the SAFE programme. ${ }^{17}$ Quantitative data were collected from the 12 hospital sites participating in Wave 1 of the SAFE programme and qualitative data (including observations of huddles and interviews with hospital staff about their experiences of implementing SAFE) were collected from four of these sites. The four sites were sampled for their heterogeneity of clinical context, aiming for maximal variation in terms of type of work done on the ward, size of the ward, geographical region and type of hospital. The focus of our study is on audio recordings of huddles conducted during huddle observations at these four sites. Data collection occurred 4 months after the start of SAFE (January-March 2015). All huddles that took place at the four sites for 2 days within this period were audio recorded by the evaluation team. While the purpose of a huddle is of sharing information and planning within the staff group in relation to at-risk patients and situations, SAFE sites were encouraged to implement the huddle in a contextually sensitive manner, such as to fit with their own ward structures and routines. For this reason, there was some variation in the number of huddles across the sites, the times of day at which huddles were held, huddle location and huddle attendees at each site (both in terms of numbers and staff roles, ${ }^{18}$ for further information). The frequency of the huddles across the four wards at the sites ranged from 1 to 3 per day. This provided a total of 16 huddle recordings to analyse. Huddles ranged from $1 \mathrm{~min} 40 \mathrm{sec}$ to $10 \mathrm{~min}$ in length. See table 1 for information about the sample.

\section{Patient involvement}

The SAFE collaborative ${ }^{17}$ included a parent on the planning and oversight committees and Project Board which provided insight and comment on the proposed

\begin{tabular}{|c|c|c|c|}
\hline & Type & $\begin{array}{l}\text { Number } \\
\text { of huddles } \\
\text { observed }\end{array}$ & $\begin{array}{l}\text { Transcript } \\
\text { data used in } \\
\text { analysis }\end{array}$ \\
\hline Ward 1 & $\begin{array}{l}\text { Paediatric ward } \\
\text { in a large general } \\
\text { hospital }\end{array}$ & 6 & Full \\
\hline Ward 2 & $\begin{array}{l}\text { Paediatric ward } \\
\text { with a high } \\
\text { dependency unit } \\
\text { (HDU) in a general } \\
\text { hospital }\end{array}$ & 4 & Full \\
\hline Ward 3 & $\begin{array}{l}\text { HDU ward in } \\
\text { a specialist } \\
\text { children's hospital } \\
\text { (SCH) }\end{array}$ & 4 & Partial \\
\hline Ward 4 & $\begin{array}{l}\text { General ward in a } \\
\mathrm{SCH}\end{array}$ & 2 & Partial \\
\hline
\end{tabular}


intervention and on the research undertaken. In this analysis, the focus was on staff interaction rather than the patients.

\section{Data collection}

The huddles were audio recorded by four non-participant observers, two of whom were present at any one time. The observers recorded the order of speakers to aid transcription. They completed an observational tool, specifically designed for huddles. ${ }^{19}$ Huddles were audio recorded using two recorders at opposite sides of the huddle space. The audio recordings were transcribed by observers present on the ward using simplified conversation analytic conventions. ${ }^{20}$

\section{Data analysis}

Recordings from four sites were analysed. Due to difficulties with audio sound quality, two sites, Wards 1 and 2 , provided the core material for analysis. Intelligible sections of transcripts from the other two sites were used. A researcher who was not present at data collection analysed this material. The first pass analysis was then analysed with an advisor to the project and another researcher, in which analytic disagreements were discussed and resolved. Analysis was guided by the principles of EMCA. ${ }^{21} 22$

Analysis began with the broad question of 'how do huddles happen in practice?' and through the process of examining both audio recordings and transcripts a narrower question became pertinent: how are staff identifying at-risk patients in huddles? This question was selected out of several possible phenomena for its clinical relevance and can be further broken down into:

1. What terms are staff using to categorise their patients?

2. How do they coordinate with one another in reviewing their patients?

For reasons of brevity, the focus of this paper is on question 1 but observations will also be made in relation to question 2 in the main analysis as well as in supplementary analyses. There was no fixed format for the huddle and each team had their own script and process.

The analytic steps were then to:

a. Identify all sections where a patient is identified as a risk.

b. Conduct within-case sequential analysis of the process by which at-risk patients are identified, including lexical choices and methods of implicit categorisation.

c. Conduct cross-case classification of the methods that staff used to identify at-risk patients.

\section{Reflexive statement}

Data were analysed by a researcher in the independent evaluation of the SAFE programme, not invested in the outcome of individual huddles nor the SAFE programme.

\section{ETHICAL CONSIDERATIONS}

All identifying details (including names of participants, patients and places) were disguised or removed in the transcripts of the huddle recordings. Any member of staff who did not wish to be recorded was given the opportunity to opt out prior to the recording beginning. There were no opt outs at any recording session.

\section{RESULTS}

\section{How are at-risk patients identified in huddles?}

There were four key terms used to identify patients as well as some use of implicit categorisation. Four extracts are given to illustrate the emerging lexicon with a further three in supplementary analyses (see the online appendix), as well as how this was used by the staff present.

\section{'No concerns' and 'pre-concerns'}

Huddlers displayed ways of showing for each other which patients were at risk. Sometimes identification was by making lexical choices to label patients, and sometimes potentially at-risk patients were identifiable through a lack of categorisation-for patients who were not in need of further attention the nurses used the phrase no concerns. Extract 1 taken from Ward 1 exemplifies one way in which this occurred.(box 1 )

After the ward manager opens the meeting, Nurse 1 self-selects and makes her categorisation, no concerns (line 2), providing a brief report ('Pewsing one....'; line $2)$. The doctor shows receipt of this information and then prompts the next turn, using the patient's name. Nurse 1 offers the categorisation no concerns (line 7) in response, without expansion. Many in the room coordinate at lines 9 and 10 to prompt the next speaker. Nurse 2 then does not begin her turn by offering a categorisation. She instead provides a report on the patient's situation. Nurse 1's closed question at line 13 ('Concerns or no [concerns?]') implies that this lack of categorisation is problematic. The question suggests both that the most relevant action here is a categorisation, and that it is Nurse 2 who is best placed to make it (no other medical professionals in the room are asked). After the prompting to categorise by Nurse 1 at line 13, Nurse 2's phrasing 'at the moment' (line 14) highlights the time-bound nature of her concern-in the 'moment' of this huddle, the patient is not deteriorating, but she hints that change is possible. Arguably, it introduces a third category, the concerns/the no concerns and those somewhere between the two. If concerns are anticipations of risk or deterioration, then this third category represents an anticipation of concerns - these might be termed pre-concerns. This could be viewed as a superordinate level of SA. But whether this level has a place here is for the huddle to decide. The continuation from line 15 of their previous turn taking indicates that this is enough discussion of this patient for now.

This brief exchange highlights something important about huddles. In theory, huddles are places where potential risks and concerns are discussed, but in a 'rapid exchange'. There is a necessary tension between looking ahead, and expediency and efficiency-Nurses 1 and 2 personify this tension here. In this huddle, the 
Box 1 Extract 1: Ward 1, day 2, evening

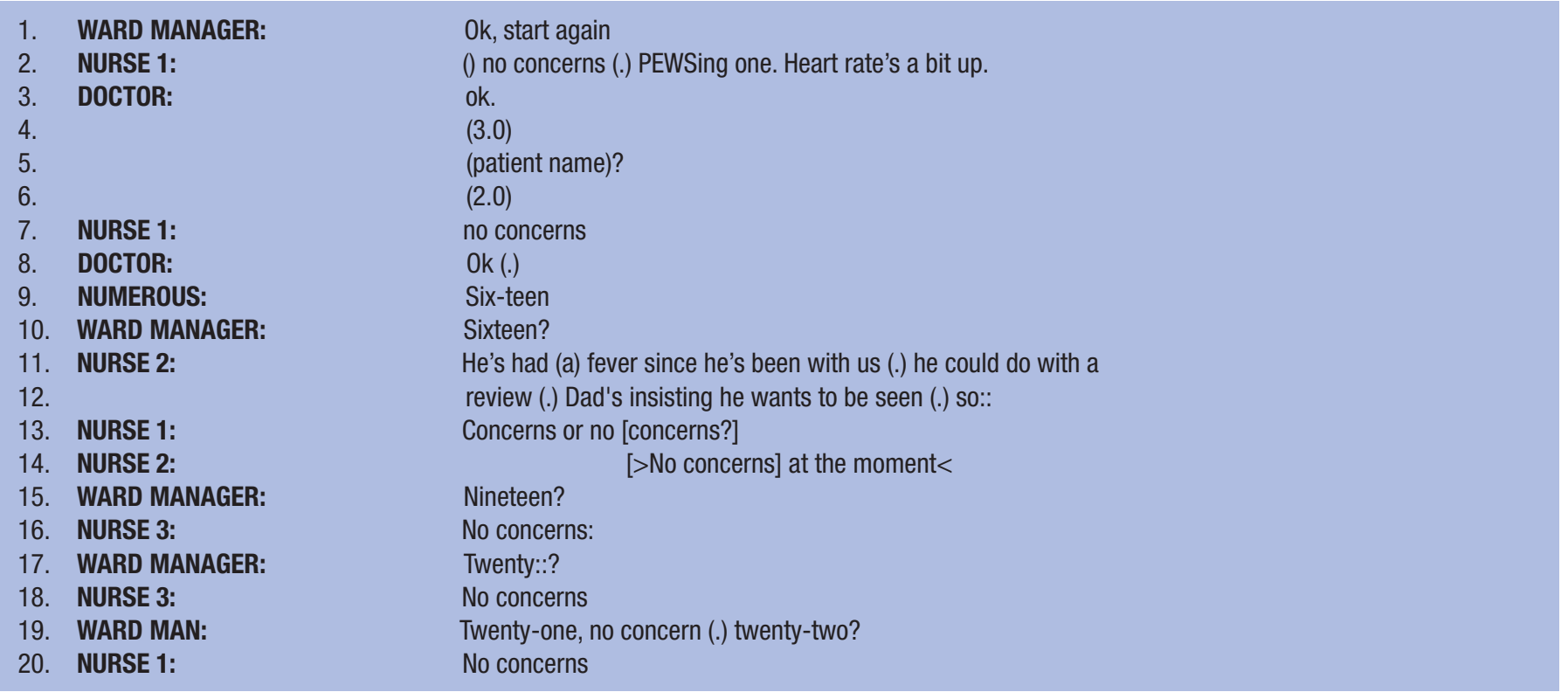

ward manager and then nurses took the lead, the doctor only becoming involved and then planning based on the clinical information, when reports were given. Nurses were responsible for bringing the right information to the huddle and classifying patients, but if the categorisation was ambiguous, this was where the doctor became involved (not seen in this extract).

A second method that huddlers used to identify at-risk patients may be seen in extract 2. (box 2) In this extract, from Ward 2, staff also used the term 'concern' but the process through which patients were identified was quite different.

In this huddle, after the consultant (attending or senior physician) opens the meeting (line 10), the staff nurse gives a general gloss: 'we're not concerned about anybody' (lines 12-14). She then unpacks this. This is different to the method of huddling where each patient is discussed in turn, and where bedside nurses each have a slot to talk. In extract 2, the staff nurse curiously demonstrates her lack of concern about the PEWS in the 'amber range'. The consultant's addition of 'we had six... it's now four' (line 20) provides the rationale for this lack of worry, as this indicates improvement. (The PEWS, or Paediatric Early Warning System includes a score which aims to be a standardised measure of the clinical state of paediatric patients. Patients are rated on cardiovascular, respiratory and behavioural vital signs and given a score, or alternatively may follow a tracker system. There are several types of PEWS (for a review, see 23)).

\section{'The one to watch'}

Extract 3 shows a sequence toward the beginning of a huddle, where the senior nurse, who is the assigned huddle leader, is 'interviewing' the consultant about the risks that he perceives. (box 3 )

The senior nurse opens with her question about who 'we're worried about?' (line 17). This frames the risk as a shared worry, but it is clear from the ensuing turns that it is the consultant's worries that are relevant; there are two other doctors present as an audience, and this huddle proceeds as an exchange between the senior nurse and

\section{Box 2 Extract 2: Ward 2, day 2, evening}

\begin{tabular}{|c|c|c|}
\hline $\begin{array}{l}10 . \\
11 .\end{array}$ & CONSULTANT: & $\begin{array}{l}\text { (Shall we do the) board huddle? } \\
(3.0)\end{array}$ \\
\hline 12. & STAFF NURSE: & Okay: so:: we've (.) ehm, [we're not] \\
\hline 13. & CONSULTANT: & {$[0]$} \\
\hline 15. & CONSULTANT: & $\mathrm{OK}=\mathrm{ehr}=$ \\
\hline $\begin{array}{l}16 . \\
17 .\end{array}$ & STAFF NURSE: & $\begin{array}{l}=\text { we've got- one HDU patient, (patient's name) who } \\
\text { is PEWing at four[0] }\end{array}$ \\
\hline 19. & STAFF NURSE: & Yeah \\
\hline 20. & CONSULTANT: & So we had six in the morning, so it's now four [so it's improving] \\
\hline 21. & STAFF NURSE: & [Hmm yes] \\
\hline
\end{tabular}




\section{Box 3 Extract 3: Ward 3, day 2, afternoon}

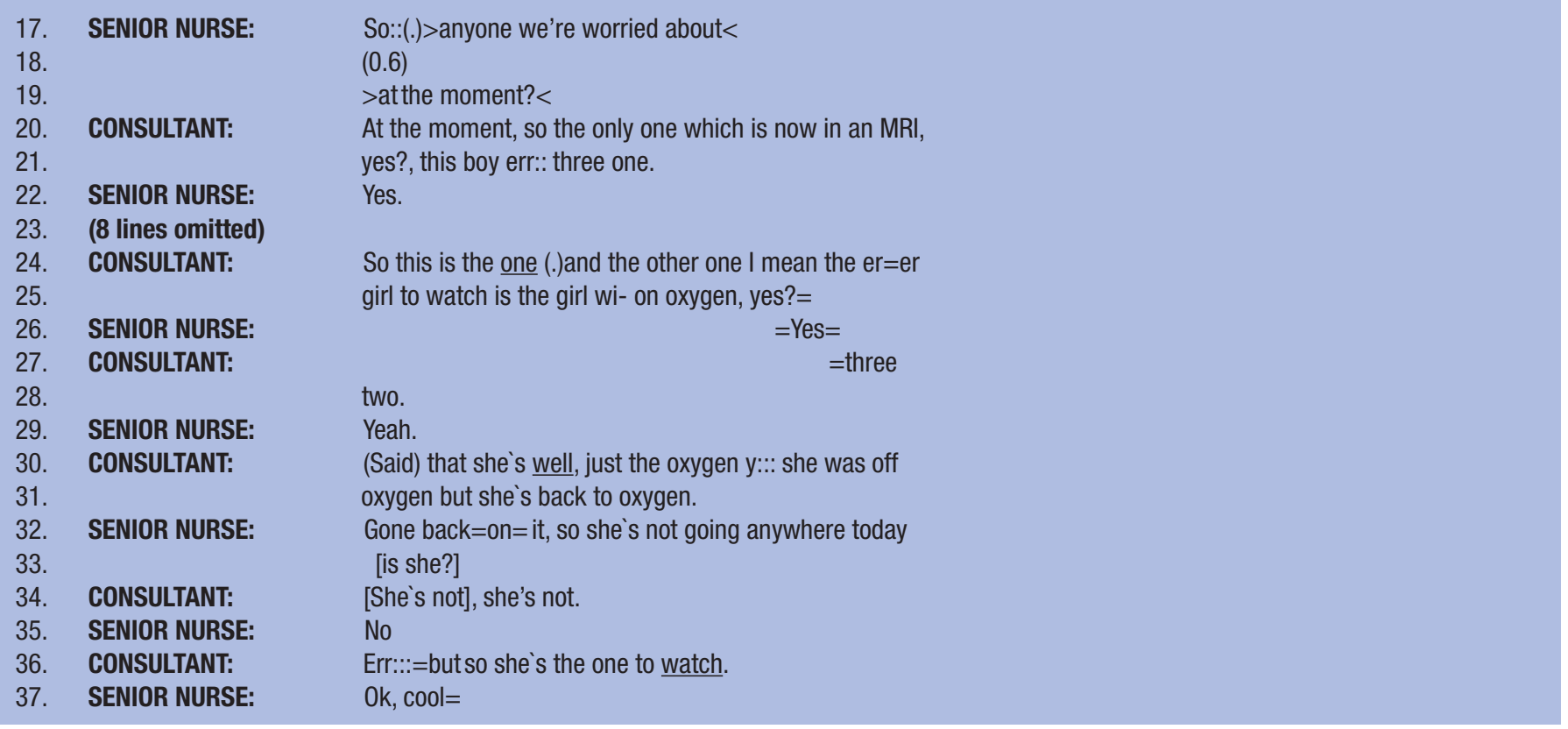

the consultant, with no 'slots' provided to other members of the team to relay information. The consultant responds to the senior nurse's opening question by talking about two patients. He marks the first patient as 'the only one' (line 20) that they are worried about, but then this 'one' is joined by another patient at lines 24-25. He makes salient that this second patient is the girl to watch'. This phrase, which uses the infinitive form of the verb 'to watch' (line 36) alongside the subject ('the one'), locates this quality of risk within the patient rather than in the feelings (ie, 'concerns') of the clinician. It also has a plan embedded within it-'to watch' them, to be more aware of them. The use of the infinitive form means that this could be a general instruction to all at this huddle or for the senior nurse. The senior nurse accepts the consultant's assessment of the situation with the 'ok, cool' (line 37) but there is no verbal input from the others present.

In this huddle, it was very clear who the 'at-risk' patients are, and the meeting was rapid and tightly focused around them. There was no 'noise' to filter about non-risks. However, this tight focus seemed to be at the expense of collaboration, in the sense that huddles on this ward were organised around one person's perception of risk.

\section{'The watchers'}

At Cincinnati Children's Hospital, staff use the phrase 'watcher' as a noun, to discuss at-risk patients. ${ }^{3}$ We have seen how a variation of this ('the one to watch') is used to categorise patients on Ward 2. The original term, watcher, was used in huddles on Wards 3 and 4 as indicated in extract 4. (box 4)

The nurse here uses 'watchers' (line 27) in a similar way as 'the one to watch' was used at Ward 2, insofar as it quickly designates a patient as needing extra attention. However, this is more a report for the doctor that she is speaking to (this is a two person huddle) than an instruction, as 'we're keeping an eye out' (line 27) suggests that the matter is already in hand. The term watcher locates the quality of risk within an individual patient, unlike the terms 'concern' or 'worry', which foreground the feelings of a clinician. However, what all these terms have in common in terms of their function is that they are quick ways of directing the 'gaze' of the ward.

\section{DISCUSSION}

These data were taken from the early implementation phase of the SAFE programme, and it was clear that huddlers had established different methods for identifying risks to their patients. Attention to the language revealed that all wards had adopted terms to establish shared concerns under time pressure. Teams varied in the way patients were identified. The first method was to identify patients one by one as in excerpt 1 . In this method, a senior member of staff (doctor or nurse manager) names

\section{Box 4 Extract 4: Ward 4, day 1, morning}

26. NURSE: $\quad$ No cardiac arrests respiratory arrests, PICU admissions. Erm,
27.
28.
thewatchers, is (patient name) we're keeping an eye out, and 24


the patient, thereby soliciting a categorization, and the nurse procures it (method 1a, excerpt 1 ). An alternative was for the nurse to name the patient and then categorises them (method $1 b$, excerpt 5 in online appendix). The second method was to identify problem patients as in excerpt 2. In this method, a senior member of staff sometimes solicits talk about problematic cases (method 2a, excerpt 3), and sometimes the nurse him/herselfintervenes (method 2b, excerpt 2).

The development of similar 'reliable flagging processes' was found to be important in alerting a team to where to focus their attention overnight in a study of 'hospital at night handovers' ${ }^{24}$ Huddlers showed adaptation of their terms in situations where the patient resisted simple categorisation. As with Eggins and Slade ${ }^{16}$ analysis showed the sensitivity of huddlers to what Maynard and Heritage $^{25}$ have termed 'socio-medical' dilemmas, in other words the interdependence of information sharing with social interaction that is broadly cooperative.

Despite the variety in lexical choice and processes of identifying risk, one common thread was the characteristics of the concerns and risks discussed, in that they were all situations that required measures outside the 'business as usual' practices of the ward. This meant that the huddlers' understandings of risk were in part, locally defined. For example, an ill patient with a certain condition on one ward may have been a concern, yet on another they may have been a typical patient. Moreover, risks to patients were time bound, so that a high PEW score was not seen as a concern if the score was lower than the previous huddle. There was a necessary element of: (1) ward centredness and (2) patient centredness, in definitions of risk, and this shows a need to go beyond standardised tools as standalone indicators of risk. Risks were conversationally negotiated, and this conversation was inherently continuous with previous huddles.

When someone raised a concern, there were various choices that could be made by other huddlers, either to facilitate the speaker to say more, to prompt them to categorise the patient, or to close the topic down and move on. There were also implicit rules in operation about the conversational roles of huddlers-both in terms of managing the trajectory of the talk, and the epistemic realms that different staff roles exercised. Although this varied considerably across huddles, there was also some stability within wards. For example, in Ward 1, only bedside nurses gave information about patients, and they were 'interviewed' by the other members of the team. This implies that they had the epistemic authority to offer the best information. However, doctors had to agree that a situation was sufficiently concerning to require a plan-therefore, doctors made or confirmed the final assessment on a patient and made moves to close topics. In others, Ward 3, for example, the consultant identified the risks by providing information, as well as closing topics and moving to new topics-it was the senior nurse that showed receipt of this information. The consultant

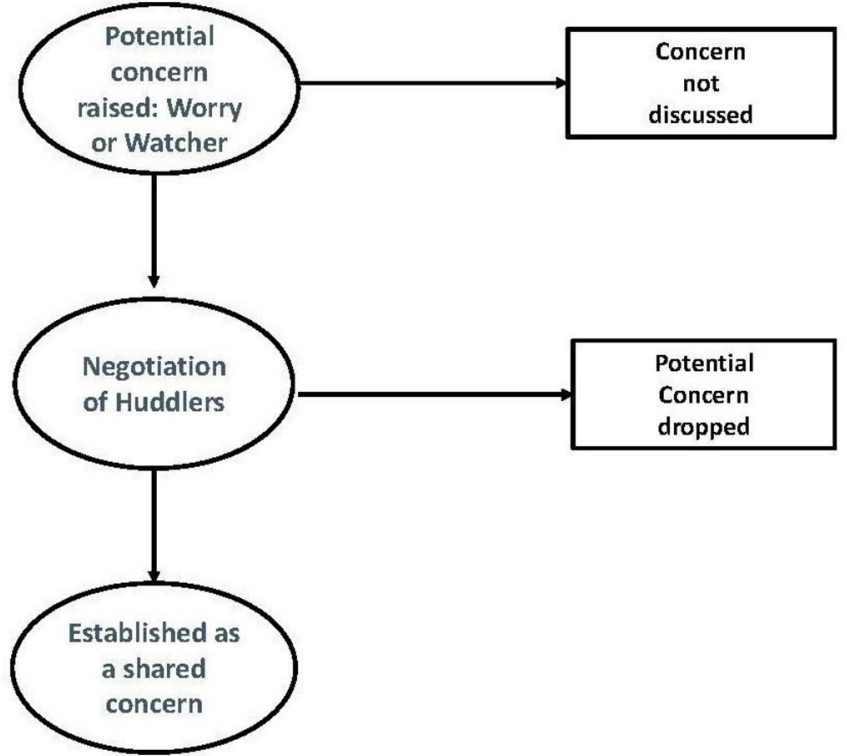

Figure 1 Methods used to establish shared concerns.

exercised a larger range of conversational moves and epistemic realms.

Huddlers usually do not talk about patients as 'really ill/poorly/sick', and this is because ill patients are not concerning to them if they are stable, and if their needs are within the bounds of current institutional processes. Instead, huddlers needed, and are developing, other terms that can capture not simple static states but changes, and potential changes-labels that index the past, present and future. The other thing to note is that concerns and risks that are raised by someone in a huddle need to go through a process to become established by the huddle as a shared problem, and that this process may be more, or less collaborative. The speaker first needs to be given the floor for long enough to offer all relevant information. Second, this information needs to be considered by the senior staff present. Although each huddle was different, there were some general features of organisation that huddlers used to discuss potential risks to patients (figure 1).

\section{PRACTICAL IMPLICATIONS AND RECOMMENDATIONS}

\section{To discuss the non-concerns?}

Some huddlers spoke only about situations that concerned them and others used the time to speak briefly about each patient bed. The advantage of this latter method of huddling was that there were 'slots' created for nurses in the huddle to communicate potential changes in patients. ${ }^{13}$ Due to the tensions between providing opportunities for collaboration and expediency, huddlers may reflect on the best use of their time.

\section{Language}

We noted the different terms that huddlers used and as with all language it is not simply what word that is used but also how it is used that is important. One consideration is 
the meaning and function that these terms had in this sample. Watchers was used to speak only of at-risk patients, rather than other problematic situations. This included the sharing of 'gut feelings'-when there were no clinical indicators of risk, but where someone senses something is wrong. The one to watch is used similarly but more explicitly contains instruction. Concerns were used to talk about patients but also were used to index other problems. There were also situations that resisted simple categorisation and were termed by the authors preconcerns. Huddlers may consider having a category that captures such pre-concerns or 'pre-watchers'.

\section{Roles}

Senior staff members were the most active in channelling the talk in huddles. For example, in asking questions, and using 'continuers' when others provided information. In some huddles, only senior staff shared their knowledge and concerns about patients. Is this situation desirable? Huddle theoreticians and practitioners could reflect on whether the most junior members of staff should have a greater role in huddles.

\section{Enabling the communication of concerns}

Communicating information about a patient is an important element of SA, but equally important is how the listening happens. Analysis showed that the use of various response tokens and questions, channelled speakers to provide information on patients or close the topic. It is recommended that huddlers consider the ways that they encourage others to speak and share concerns and display that these are taken seriously.

\section{LIMITATIONS}

The data reported here were derived from the early implementation of huddles and it is possible that over time, the variety of methods that members used in the current analysis may change with growing experience. The quality of the data used was not consistent due to recording problems at two of the sites and this constrained a more detailed sequential analysis. Use of video data was not possible in this project due to the ethical sensitivity of collecting data on an open ward environment. This poses a limitation considering recommendations for multimodal analysis of meetings. ${ }^{5}$

\section{CONCLUSIONS}

The aim of this article was to highlight how healthcare staff members translate huddle theory into practice, and it is the first study to examine the discourse of huddles. It has been found that specific lexical markers are in use at all wards, and that these allow the expedient identification of patients who are at risk of deterioration. Huddlers also adapted these terms to both upgrade and downgrade risk, suggesting that standardised indicators of risk were not enough alone for defining risks.
Sequential analysis has also highlighted the conversational rights held implicitly by staff in different roles. This has displayed a potential tension between huddle principles and the fact that the more senior staff in these huddles seemed to be using the greatest variety of conversational moves. Findings may aid huddlers in considering the ways of conversing that best promote huddle principles on their ward.

\section{Author affiliations}

${ }^{1}$ Psychology, University of Roehampton, London, UK

${ }^{2}$ Executive Department, International Society for Quality in Healthcare (ISQua), Dublin, Ireland

${ }^{3}$ Quality Improvement, Royal College of Paediatrics and Child Health, London, UK ${ }^{4}$ Evidence Based Practice Unit, University College London and the Anna Freud Centre, London, UK

${ }^{5}$ Evidence Based Practice Unit, Anna Freud National Centre for Children and Families, London, UK

${ }^{6}$ Department of Clinical, Educational and Health Psychology, University College London, London, UK

${ }^{7}$ CAMHS Evidence Based Practice Unit, UCL and Anna Freud Centre, London, UK

Acknowledgements The authors would like to thank Dawid Gondek, Amy Ramsay, Evelyn Sharples and Makeda Gerressu for their role in data collection and transcription. Sincere thanks are also extended to Ivan Leudar, and the Roehampton EMCA group for analytic wisdom; and Sarah Cantwell, John Rae, and Adam Gibson for constructive comments on an earlier draft. The authors thank their Patient Advisor Emma Francis for her valuable input to the SAFE programme.

Contributors $\mathrm{JH}$ led the ECMA research and drafting of the paper under the supervision of JD and MW, and $\mathrm{JH}$ conducted the literature review and developed the paper. JH and PL wrote the final version of the paper. ES provided revision of the paper. JE-C contributed to analyses. All authors contributed to the drafting of the paper.

Funding Situation Awareness For Everyone (SAFE) is a Health Foundation funded programme; both the implementation of SAFE and the evaluation were funded by the Health Foundation. This work was also supported by funding from WellChild, the funding was specifically to support evaluation work around the perspectives of parents and young people and to support patient and parent involvement in the research. This programme of work and evaluation was also supported by the Royal College of Paediatrics and Child Health, which leads on the delivery of the programme. JD was supported by the National Institute for Health Research Collaboration for Leaderships in Applied Health Research and Care North Thames at Bart's Health NHS Trust.

Disclaimer The views expressed are those of the authors and not necessarily those of the NHS, the NIHR, the Department of Health, or RCPCH.

Competing interests PL led the implementation of the SAFE programme and contributed to the literature review and drafting of this manuscript. However, PL was not involved in the data collection nor data analysis/results reported here.

Patient consent for publication Not required.

Ethics approval Dulwich Research Ethics Committee (REC reference: 14/ LO/0875).

Provenance and peer review Not commissioned; externally peer reviewed.

Data sharing statement This is part of a larger study and three papers have already been published. This paper is a specific analysis and does not compete with other data held. All data are held by the research team at the Anna Freud Centre. Supporting data and analyses are available in the appendices. Additional data are available on request to the authors.

Open access This is an open access article distributed in accordance with the Creative Commons Attribution Non Commercial (CC BY-NC 4.0) license, which permits others to distribute, remix, adapt, build upon this work non-commercially, and license their derivative works on different terms, provided the original work is properly cited, appropriate credit is given, any changes made indicated, and the use is non-commercial. See: http://creativecommons.org/licenses/by-nc/4.0/. 


\section{REFERENCES}

1. Brady PW, Muething S, Kotagal U, et al. Improving situation awareness to reduce unrecognized clinical deterioration and serious safety events. Pediatrics 2013;131:e298-308.

2. Goldenhar LM, Brady PW, Sutcliffe KM, et al. Huddling for high reliability and situation awareness. BMJ Qual Saf 2013;22:899-906.

3. Provost SM, Lanham HJ, Leykum LK, et al. Health care huddles: managing complexity to achieve high reliability. Health Care Manage Rev 2015;40:2-12.

4. RCPCH. Situation Awareness for Everyone (S.A.F.E) Programme. http://www.rcpch.ac.uk/safe (Accessed 8th Nov 2016).

5. Deppermann A, Schmitt R, Mondada L. Agenda and emergence: contingent and planned activities in a meeting. J Pragmat 2010;42:1700-18.

6. Housley W. Role as an interactional device and resource in multidisciplinary team meetings. Sociol Res Online 1999;4:1-14.

7. Huisman M. Decision-making in meetings as talk-in-interaction. International Studies of Management \& Organization 2001;31:69-90.

8. Caronia L, Saglietti M. Knowledge and agency in interprofessional care: How nurses contribute to the case-construction in an Intensive Care Unit. J Interprof Care 2018;32:592-602.

9. Izumi H. Help-search practices in rehabilitation team meetings: a sacksian analysis. Hum Stud 2017;40:439-68.

10. Albury C, Stokoe E, Ziebland S, et al. GP-delivered brief weight loss interventions: a cohort study of patient responses and subsequent actions, using conversation analysis in UK primary care. $\mathrm{Br} J \mathrm{Gen}$ Pract 2018;68:e646-e653.

11. Heritage J, Maynard DW. Communication in medical care. Cambridge, England: Cambridge University Press, 2006.

12. Heritage J, Robinson JD. The structure of patients' presenting concerns: physicians' opening questions. Health Commun 2006;19:89-102.

13. Chatwin J, Kennedy A, Firth A, et al. How potentially serious symptom changes are talked about and managed in COPD clinical review consultations: a micro-analysis. Soc Sci Med 2014;113:120-36.

14. Stivers T. Participating in decisions about treatment: overt parent pressure for antibiotic medication in pediatric encounters. Soc Sci Med 2002;54:1111-30.

15. Raduma-Tomàs MA, Flin R, Yule $S$, et al. Doctors' handovers in hospitals: a literature review. BMJ Qual Saf 2011;20:128-33.

16. Eggins S, Slade D. Clinical handover as an interactive event: informational and interactional communication strategies in effective shift-change handovers. Commun Med 2012;9:215-27.

17. Deighton J, Edbrooke-Childs J, Stapley E, et al. Realistic evaluation of Situation Awareness for Everyone (SAFE) on paediatric wards: study protocol. BMJ Open 2016;6:e014014.

18. Stapley E, Sharples E, Lachman P, et al. Factors to consider in the introduction of huddles on clinical wards: perceptions of staff on the SAFE programme. Int J Qual Health Care 2018;30:44-9.

19. Edbrooke-Childs J, Hayes J, Sharples E, et al. Development of the Huddle Observation Tool for structured case management discussions to improve situation awareness on inpatient clinical wards. BMJ Qual Saf 2018;27:365-72. Sep 19.

20. Jefferson G. Glossary of transcript symbols with an introduction. Lerner $\mathrm{GH}$, ed. Conversation analysis, studies from the first generation. Amsterdam, Netherlands: John Benjamins, 2004:13-31.

21. Garfinkel H. Studies in ethnomethodology. Cambridge: Prentice Hall, 1967.

22. Sacks H. Lectures on conversation. Oxford, England: Blackwell, 1992.

23. Lambert $\mathrm{V}$, Matthews $\mathrm{A}$, MacDonell $\mathrm{R}$, et al. Paediatric early warning systems for detecting and responding to clinical deterioration in children: a systematic review. BMJ Open 2017;7:e014497.

24. McQuillan A, Carthey J, Catchpole K, et al. Republished: creating a safe, reliable hospital at night handover: a case study in implementation science. Postgrad Med J 2014;90:493-501.

25. Maynard DW, Heritage J. Conversation analysis, doctor-patient interaction and medical communication. Med Educ 2005;39:428-35. 